\title{
Endoscopic dissection of an esophageal submucosal tumor using a novel bipolar radiofrequency device
}

A 42-year-old woman was diagnosed with a submucosal lesion at the lower esophagus extending into the fundus ( $\mathbf{F i g . 1}$ ). Endosonography showed the tumor arising from the second layer (i. e. muscularis mucosa). Submucosal tunneling endoscopic resection (STER) was performed using a therapeutic channel (3.7 mm) endoscope (GIF-1TH190; Olympus, Tokyo, Japan) ( $\vee$ Video 1 ). We used a new multimodality knife for the entire procedure (Speedboat-RS2; Creo Medical Ltd., Chepstow, UK) (ฉ Fig. 2). This device consists of curved bipolar electrodes on the sides for cutting using radiofrequency energy $(400 \mathrm{kHz}$ and $35 \mathrm{~W})$ and microwave for coagulation (frequency 5.8 $\mathrm{GHz}$, power setting $10 \mathrm{~W}$ ). In brief, the STER procedure involved: a) submucosal injection at about $1 \mathrm{~cm}$ proximal to the submucosal tumor ( Fig.3a); b) mucosal incision of about $2 \mathrm{~cm}$ in length ( Fig.3b); c) submucosal tunneling and dissection of the tumor from surrounding tissue ( $\vee$ Fig. $3 \mathbf{c}$ ); d) retrieval of the tumor using a polypectomy snare ( $\vee$ Fig. 3 d); d) closure of the incision using multiple endoclips.

The tunneling technique is widely utilized for the resection of submucosal tumors in the upper gastrointestinal tract. Advances in devices and techniques have improved the outcomes of endoscopic resection in these lesions [1]. This case demonstrates the use of a new multimodality device for endoscopic dissection of a large submucosal tumor. The novel bipolar cutting device has an integrated injection needle so that the entire procedure can be accomplished without device exchange. The presence of a protective hull ( $>$ Fig. 2) safeguards against inadvertent damage to the muscle, which is a potential concern when using monopolar electrosurgical knives [2]. A recent report

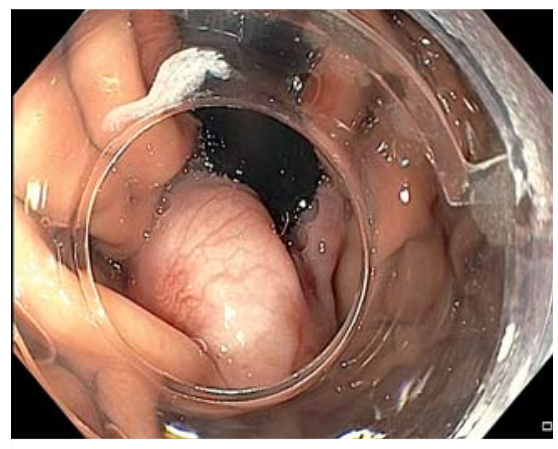

- Fig. 1 Endoscopic image revealing a globular submucosal lesion in the fundus.

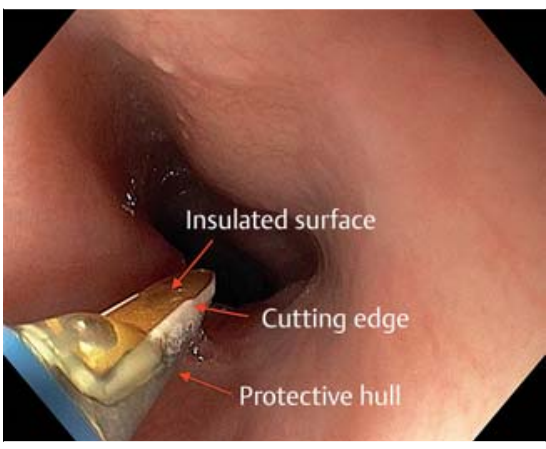

Fig. 2 Novel multimodality device used for performing endoscopic resection of the submucosal tumor.
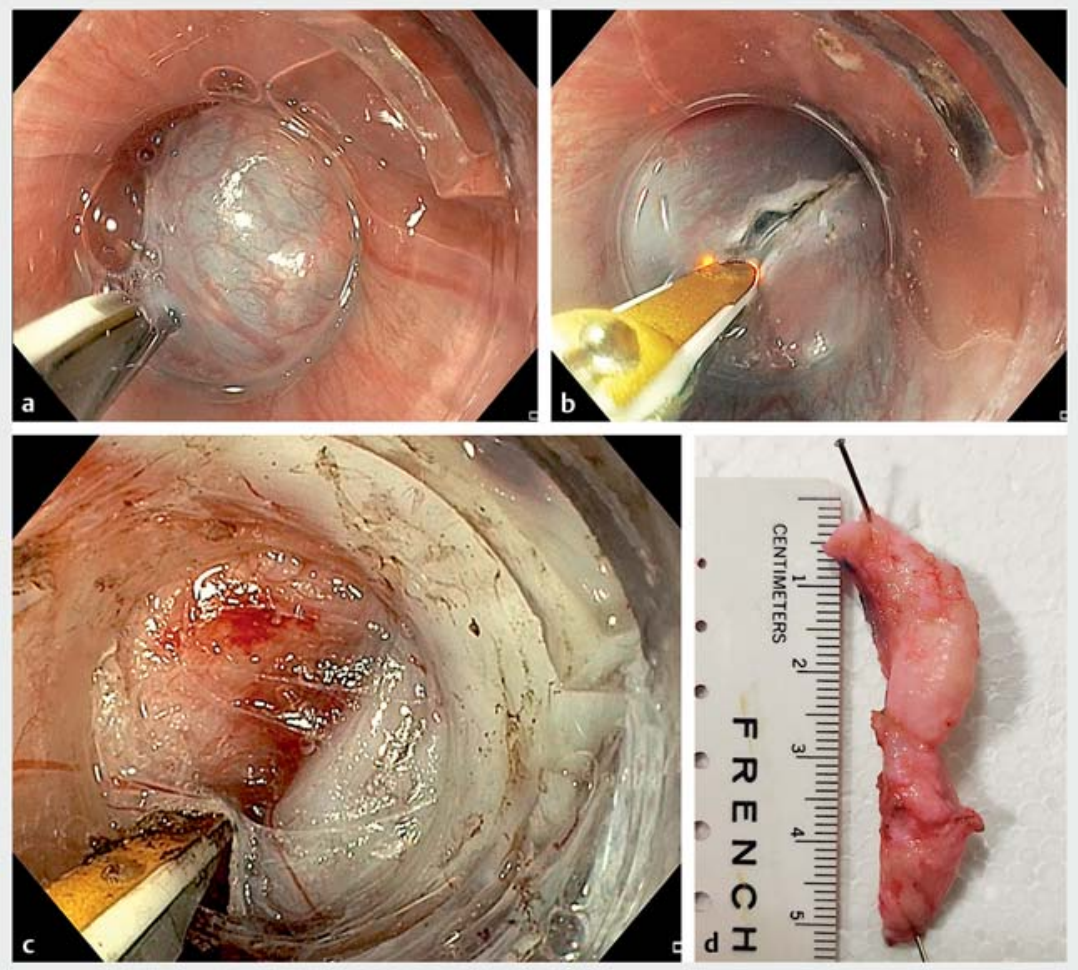

- Fig. 3 The submucosal tunneling endoscopic resection procedure. a Submucosal injection with indigo carmine diluted with saline. $\mathbf{b}$ Mucosal incision above the tumor using the same bipolar knife. c Dissection of the tumor from the surrounding tissue. $\mathbf{d}$ Resected submucosal tumor. 


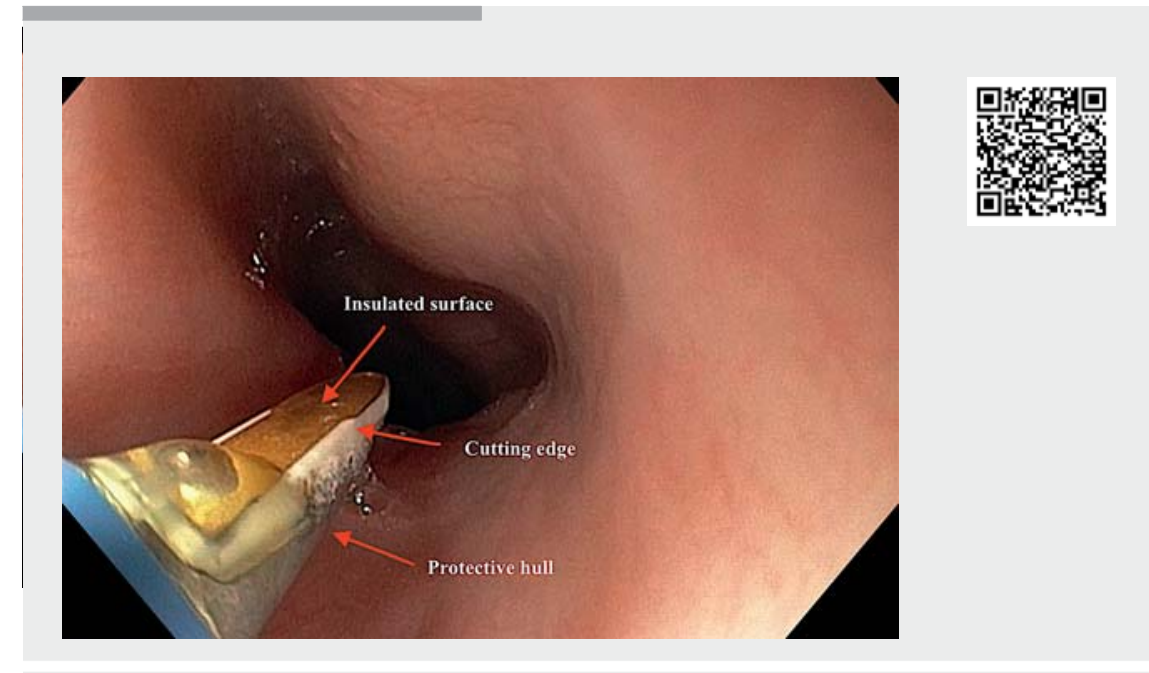

$\checkmark$ Video 1 Endoscopic resection of a large esophageal submucosal lesion using a novel, bipolar, multimodality device.

described the use of this device for removal of a large colonic polyp by the endoscopic submucosal tunneling dissection technique, with no muscle damage and only minimal charring [3].

\section{Endoscopy_UCTN_Code_TTT_1AO_2AG}

\section{Competing interests}

The authors declare that they have no conflict of interest.

Zaheer Nabi ${ }^{1}$, Mohan Ramchandani ${ }^{1}$, Radhika Chavan ${ }^{1}$, Santosh Darisetty ${ }^{2}$, Rama Kotla ${ }^{1}$,

D. Nageshwar Reddy ${ }^{1}$

1 Gastroenterology, Asian Institute of Gastroenterology, Hyderabad, India

2 Anesthesiology, Asian Institute of Gastroenterology, Hyderabad, India

\section{Corresponding author}

\section{References}

[1] Nabi Z, Ramchandani M, Chavan R et al. Outcome of peroral endoscopic myotomy in achalasia cardia: experience with a new triangular knife. Saudi J Gastroenterol 2018; 24: $18-24$

[2] Nabi Z, Reddy DN, Ramchandani M. Adverse events during and after per-oral endoscopic myotomy: prevention, diagnosis, and management. Gastrointest Endosc 2018; 87: 417

[3] Tsiamoulos ZP, Sebastian J, Bagla N et al. A new approach to endoscopic submucosal tunneling dissection: the "Speedboat-RS2" device. Endoscopy 2019; 51: E185-E186

\section{Bibliography}

DOI https://doi.org/10.1055/a-1089-7680

Published online: 29.1.2020

Endoscopy 2020; 52: E257-E258

(c) Georg Thieme Verlag KG

Stuttgart · New York

ISSN 0013-726X

\section{Zaheer Nabi, MD}

Asian Institute of Gastroenterology, 6-3-661, Somajiguda, Hyderabad - 500 082, India Fax: +91-40-23324255

zaheernabi1978@gmail.com 